\title{
Dangerous Liaisons? Gastroesophageal Reflux Disease and Organizing Pneumonia
}

\author{
Fotios Drakopanagiotakis \\ Second Department of Internal Medicine, Justus Liebig University Giessen, Giessen, Germany
}

In the last issue of Respiration, Gaillet et al. [1] report on the significance of gastroesophageal reflux disease (GERD) as a risk factor for the severity of organizing pneumonia (OP). In their series, patients with OP and GERD had more extensive radiological involvement, greater hypoxemia and greater bronchoalveolar lavage cellularity compared to those with OP in the absence of GERD. Moreover, relapses occurred more often in the group with than that without GERD. An association between GERD and lung diseases has long been suggested [2-4].

GERD is a well-recognized cause of posterior laryngitis and chronic cough $[5,6]$, and a high prevalence of GERD has also been reported among patients with asthma [7]. In patients with lung fibrosis due to scleroderma, GERD is a common co-diagnosis and the severity of aspiration is associated with a worse prognosis [8]. Following lung transplantation, the aspiration of gastrointestinal contents has also been linked to a worse outcome and the development of bronchiolitis obliterans [9]. GERD is considered a risk factor for idiopathic pulmonary fibrosis (IPF). Animal studies, a prevalence of GERD reaching $94 \%$ in IPF patients [10] and a more favorable disease course after GERD treatment support this concept [11].

How does GERD contribute to the development of parenchymal lung disease? The main pathogenetic mecha- nism is chronic recurrent subclinical microaspiration of gastric content or foreign particles into the lungs [2]. Although normal individuals aspirate small amounts of oral secretions during sleep [12], a more prolonged aspiration can lead to the development of parenchymal lung disease. Gastric and bile acids have been shown to elicit an inflammatory response in the lung, and food particles per se, at a neutral $\mathrm{pH}$, can also be the cause of lung inflammation leading to the accumulation of giant cells and the development of granulomas [3]. Although GERD does not necessarily lead to aspiration or microaspiration [13], patients with advanced fibrotic lung diseases (IPF and pretransplant/scleroderma-associated fibrosis) have more often and more severe GERD [14, 15].

$\mathrm{OP}$ is characterized by a pathological pattern of patchy filling of the lung alveoli and respiratory bronchioles by loose plugs of granulation tissue. As a clinical entity, OP is associated with nonspecific symptoms of cough and dyspnea and radiographic findings of patchy alveolar infiltrates. OP may be associated with other underlying diseases (secondary OP) or it may be idiopathic (cryptogenic OP). Contrary to a fibrosing process such as in usual interstitial pneumonia/IPF, it responds strikingly well to corticosteroid therapy. On other occasions, OP may be just an incidental finding in lung biopsy specimens or
Dr. Fotios Drakopanagiotakis

Medizinische Klinik und Poliklinik II, Second Department of Internal Medicine Justus Liebig University Giessen Klinikstrasse 33, DE-35392 Giessen (Germany)

E-Mail Fotios.Drakopanagiotakis@ innere.med.uni-giessen.de 
may be found adjacent to lung areas affected by other diseases. This remarkable heterogeneity of OP (cryptogenic, secondary or just a pathological finding) indicates a specific histological pattern induced by a large variety of hazardous stimuli [16-18].

The association between GERD and OP is not clear. In most OP series published, GERD is neither mentioned as a cause of secondary OP nor has it been related to an increased relapse rate [19-24]. However, there are other studies which suggest a potential link between OP and GERD. In a study of 59 patients with findings of aspiration in surgical material, the cardinal histological finding was that of bronchiolitis obliterans organizing pneumonia (BOOP). Aspiration was not suspected clinically in the majority of patients [25]. Similar findings have also been described in young patients with GERD and no symptoms of aspiration with histological findings of bronchiolocentric OP and giant cells containing material consistent with food [26]. BOOP as a manifestation of aspiration rather than rejection has also been reported after lung transplantation [27]. The study by Gaillet et al. [1] examined the association between OP and GERD more closely. The prevalence of GERD in the study population was $45 \%$, which exceeds the $20-30 \%$ seen in the general population. GERD was diagnosed clinically, and its diagnosis preceded that of OP. GERD was diagnosed if typical pyrosis existed, which responded to proton pump inhibitor (PPI) treatment, and had been further validated invasively in two thirds of the patients.

It must be stated, however, that the diagnosis of GERD or GERD-associated lung disease is not easy. Although in everyday practice symptoms and response to treatment may suggest GERD, procedures such as endoscopy, 24hour $\mathrm{pH}$ monitoring, manometry and measurements of pepsin and bile acids in bronchoalveolar lavage may be necessary to secure the diagnosis $[3,15]$. Equally important, GERD can be clinically silent: half of the patients with IPF and GERD do not report classic GERD symptoms, and this also applies to patients after lung transplantation $[10,15]$. In the study by Gaillet et al. [1], GERD was excluded based on invasive assessments only in $8 \%$ of the patients in the GERD-negative group, although none of these patients had symptoms suggesting regurgitation.

Relapses are common events in the natural course of OP, but they are not associated with increased mortality [20]. Several factors have been associated with an increased frequency of relapses in OP: hypoxemia at presentation, elevated serum glutamyl transferase and alkaline phosphatase levels, the degree of collagen fibrosis in the interstitium and delayed treatment of the first episode
$[20,24,28]$. In the study by Gaillet et al. [1], the presence of GERD was associated with more relapses, which, however, did not result in significant clinical or physiological sequelae, since the total dose of corticosteroids used, hospitalizations and respiratory failure events were similar in the two groups. The authors did not find a difference in the frequency of other relapse-associated factors between the GERD-positive and GERD-negative groups, including the degree of collagen fibrosis (although the number of biopsies available was relatively small).

Is treatment for GERD an effective treatment for lung diseases? PPIs beneficially affect chronic cough associated with GERD [5], and they have been shown to correlate with increased survival in IPF in retrospective studies [29, 30]. They do not improve asthma control in patients with poorly controlled asthma [7], however, and they may be associated with adverse effects, such as increased frequency of pneumonia [31] and earlier exacerbations in patients with cystic fibrosis [32]. Moreover, repression of the acidic component of the stomach does not exclude aspiration of nonacidic components [9]. Regarding OP, data are scarce. In an earlier report, Sadoun et al. [33] presented 5 patients with BOOP with clinical and radiological improvement after medical or surgical treatment of GERD and suggested that GERD in such patients should be actively investigated and treated. Gaillet et al. [1] report that PPIs did not seem to prevent relapse in the GERD group, although a beneficial effect was reported in patients with well-controlled GERD under treatment.

Gaillet et al. [1] provide significant information regarding the relationship between GERD and OP. Additional research seems necessary in order to better elucidate the role of GERD in the pathogenesis of OP and to determine the role of antireflux therapies in the prevention of relapses.

References

1 Gaillet G, Favelle O, Guilleminaut L, de Muret A, Lemarie E, Lecomte T, Marchand-Adam S, Diot P: Gastroesophageal reflux disease is a risk factor for severity of organizing pneumonia. Respiration 2015; 89: 119-126.

2 Morehead RS: Gastro-oesophageal reflux disease and non-asthma lung disease. Eur Respir Rev 2009;18:233-43.

-3 Allaix ME, Fisichella PM, Noth I, Mendez BM, Patti MG: The pulmonary side of reflux disease: from heartburn to lung fibrosis. J Gastrointest Surg 2013;17:1526-1535.

-4 Chang AB, Lasserson TJ, Gaffney J, Connor FL, Garske LA: Gastro-oesophageal reflux treatment for prolonged non-specific cough in children and adults. Cochrane Database Syst Rev 2011;1:CD004823. 
5 Irwin RS: Chronic cough due to gastroesophageal reflux disease: ACCP evidence-based clinical practice guidelines. Chest 2006;129(1 suppl):80S-94S.

6 Irwin RS, Madison JM: The diagnosis and treatment of cough. N Engl J Med 2000;343: 1715-1721.

7 Chan WW, Chiou E, Obstein KL, Tignor AS, Whitlock TL: The efficacy of proton pump inhibitors for the treatment of asthma in adults: a meta-analysis. Arch Intern Med 2011;171: 620-629.

8 Gyger G, Baron M: Gastrointestinal manifestations of scleroderma: recent progress in evaluation, pathogenesis, and management. Curr Rheumatol Rep 2012;14:22-29.

-9 Hartwig MG, Davis RD: Gastroesophageal reflux disease-induced aspiration injury following lung transplantation. Curr Opin Organ Transplant 2012;17:474-478.

-10 Raghu G, Freudenberger TD, Yang S, Curtis JR, Spada C, Hayes J, et al: High prevalence of abnormal acid gastro-oesophageal reflux in idiopathic pulmonary fibrosis. Eur Respir J 2006;27:136-142.

11 Raghu G: Idiopathic pulmonary fibrosis: increased survival with 'gastroesophageal reflux therapy': fact or fallacy? Am J Respir Crit Care Med 2011;184:1330-1332.

12 Gleeson K, Eggli DF, Maxwell SL: Quantitative aspiration during sleep in normal subjects. Chest 1997;111:1266-1272.

$\checkmark 13$ Songur N, Songur Y, Cerci SS, Ozturk O, Sahin U, Senol A, et al: Gastroesophageal scintigraphy in the evaluation of adult patients with chronic cough due to gastroesophageal reflux disease. Nucl Med Commun 2008;29: 1066-1072.

$\checkmark 14$ Raghu G, Meyer KC: Silent gastro-oesophageal reflux and microaspiration in IPF: mounting evidence for anti-reflux therapy? Eur Respir J 2012;39:242-245.
15 Fisichella PM, Jalilvand A: The role of impaired esophageal and gastric motility in endstage lung diseases and after lung transplantation. J Surg Res 2014;186:201-206.

16 Cottin V, Cordier JF: Cryptogenic organizing pneumonia. Semin Respir Crit Care Med 2012;33:462-475.

17 Drakopanagiotakis F, Polychronopoulos V, Judson MA: Organizing pneumonia. Am J Med Sci 2008;335:34-39.

18 Cordier JF: Cryptogenic organising pneumonia. Eur Respir J 2006;28:422-446.

19 Drakopanagiotakis F, Paschalaki K, AbuHijleh M, Aswad B, Karagianidis N, Kastanakis E, et al: Cryptogenic and secondary organizing pneumonia: clinical presentation, radiographic findings, treatment response, and prognosis. Chest 2011;139:893-900.

20 Lazor R, Vandevenne A, Pelletier A, Leclerc P, Court-Fortune I, Cordier JF: Cryptogenic organizing pneumonia. Characteristics of relapses in a series of 48 patients. The Groupe d'Etudes et de Recherche sur les Maladies 'Orphelines' Pulmonaires (GERM'O'P). Am J Respir Crit Care Med 2000;162:571-577.

21 Alasaly K, Muller N, Ostrow DN, Champion P, FitzGerald JM: Cryptogenic organizing pneumonia. A report of 25 cases and a review of the literature. Medicine (Baltimore) 1995; 74:201-211.

22 King TE Jr, Mortenson RL: Cryptogenic organizing pneumonitis. The North American experience. Chest 1992;102(1 suppl):8S-13S.

23 Costabel U, Guzman J, Teschler H: Bronchiolitis obliterans with organising pneumonia: outcome. Thorax 1995;50(suppl 1):S59-S64.

24 Watanabe K, Senju S, Wen FQ, Shirakusa T, Maeda F, Yoshida M: Factors related to the relapse of bronchiolitis obliterans organizing pneumonia. Chest 1998;114:1599-1606.

25 Mukhopadhyay S, Katzenstein AL: Pulmonary disease due to aspiration of food and other particulate matter: a clinicopathologic study of 59 cases diagnosed on biopsy or resection specimens. Am J Surg Pathol 2007;31: 752-759.
26 Barnes TW, Vassallo R, Tazelaar HD, Hartman TE, Ryu JH: Diffuse bronchiolar disease due to chronic occult aspiration. Mayo Clin Proc 2006;81:172-176.

27 Miyagawa-Hayashino A, Wain JC, Mark EJ: Lung transplantation biopsy specimens with bronchiolitis obliterans or bronchiolitis obliterans organizing pneumonia due to aspiration. Arch Pathol Lab Med 2005;129:223-226.

28 Nishino M, Mathai SK, Schoenfeld D, Digumarthy SR, Kradin RL: Clinicopathologic features associated with relapse in cryptogenic organizing pneumonia. Hum Pathol 2014;45: 342-351.

29 Lee JS, Collard HR, Anstrom KJ, Martinez FJ, Noth I, Roberts RS, et al: Anti-acid treatment and disease progression in idiopathic pulmonary fibrosis: an analysis of data from three randomised controlled trials. Lancet Respir Med 2013;1:369-376.

30 Lee JS, Ryu JH, Elicker BM, Lydell CP, Jones $\mathrm{KD}$, Wolters PJ, et al: Gastroesophageal reflux therapy is associated with longer survival in patients with idiopathic pulmonary fibrosis. Am J Respir Crit Care Med 2011;184:13901394.

- 31 Eurich DT, Sadowski CA, Simpson SH, Marrie TJ, Majumdar SR: Recurrent communityacquired pneumonia in patients starting acidsuppressing drugs. Am J Med 2010;123:4753.

32 Dimango E, Walker P, Keating C, Berdella M, Robinson N, Langfelder-Schwind E, et al: Effect of esomeprazole versus placebo on pulmonary exacerbations in cystic fibrosis. BMC Pulm Med 2014;14:21.

33 Sadoun D, Valeyre D, Cargill J, Volter F, Amouroux J, Battesti JP: Bronchiolitis obliterans with cryptogenetic-like organizing pneumonia. Demonstration of gastro-esophageal reflux in 5 cases (in French). Presse Med 1988;17:2383-2385. 